\title{
How best to design Fuzzy Sets and Systems
}

\author{
In Memory of Prof. Lotfi A. Zadeh \\ Prof Plamen Angelov, PhD, DSc, FIEEE, FIET \\ Director LIRA Research Centre (www.lancaster.ac.uk/lira), Chair of Intelligent Systems, School of Computing \\ and Communications, Lancaster University, UK \\ e-mail: p.angelov@lancaster.ac.uk
}

The fundamental shift in dealing with uncertainties [12] and computerised reasoning was made by the late Professor Lotfi Aliasker Zadeh (1921-2017) in 1965 in his seminal paper [1]. For the last over five decades the Fuzzy Sets theory has matured and was applied to a long list of applications spanning from engineering, social sciences, biology to transport, mathematics and many more. One of the developments in which Prof. L. A. Zadeh had a strong personal input is the Fuzzy rule-based (FRB) systems. Perhaps the main specific features characteristic for the fuzzy sets caused a remarkable rethinking of some postulates and established concepts can be narrowed down to the following two;

a) The partial degree of membership, satisfaction, association;

b) The duality (and, more generally, the multi-multiplicity) of association.

Throughout the years of these last five decades many problems were solved in a new way thanks to the flexibility the fuzzy sets theory offers. The role of fuzzy sets in making AI (artificial intelligence) more interpretable and explainable is undeniable. Fuzzy sets offered the opportunity to formulate and solve more realistic optimisation, decision support and control problems. They are hard to be replaced in areas such as customer preferences modelling, etc.

However, one particular area of applications attracted my attention, in particular in mid-1980s when I started my research career under the supervision of Dr. Dimitar P. Filev, FIEEE, FNAE [2], namely the issue of the design of FRB systems and the closely related to them artificial neural network systems which confluence into so called neuro-fuzzy systems around that time. Another significant milestone was reached somewhat later when Hornik [3] (1990 for neural networks) and Wu and Mendel [4] (1992 for the FRB systems), respectively theoretically proved the property of the respective systems and models to be universal approximators.

The issue of the design of fuzzy sets was traditionally related to the definition of the membership function as its descriptor [5]. This postulate was not questioned so far although in late 1980s and 1990s in addition to the traditional subjective way of designing fuzzy sets (Fig. 1) the so-called data driven design method started to be popular and was developed as well (Fig. 2).

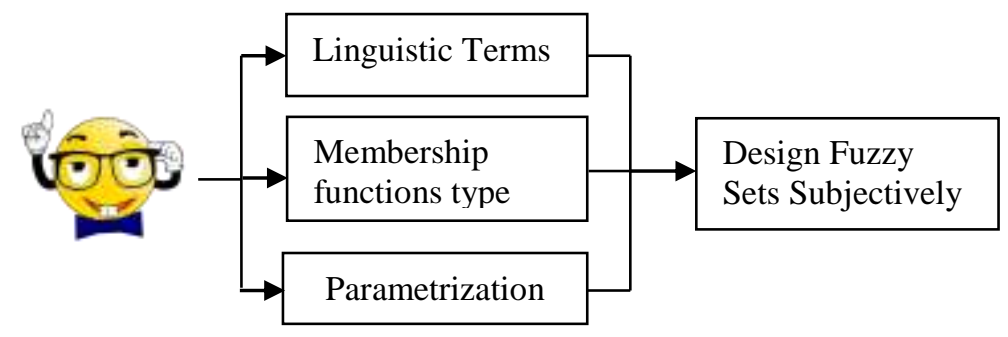

Fig. 1 Subjective design of fuzzy sets 


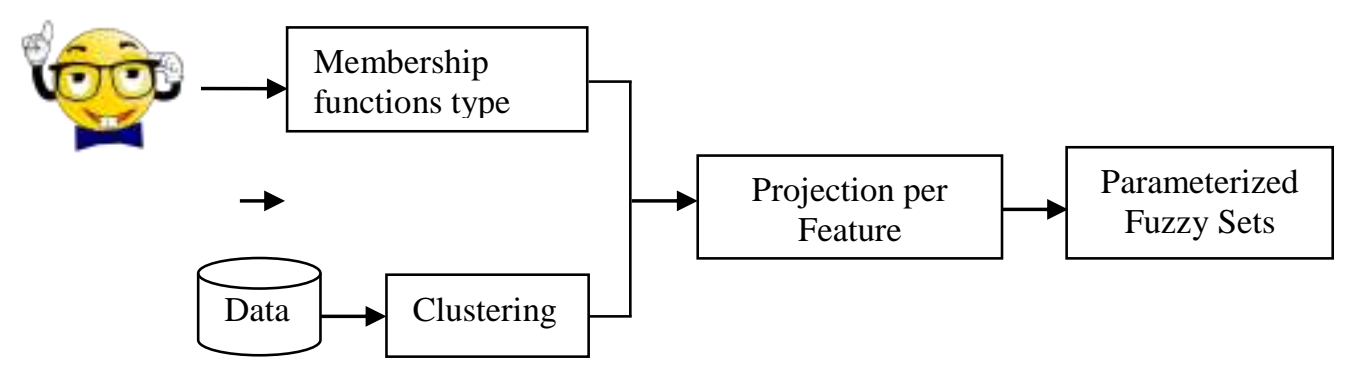

Fig. 2 Data-driven design of fuzzy sets

The subjective approach has its own very strong rationale in the two way process of:

i) extracting expert knowledge and representing it in a mathematical form through the membership functions, and

ii) the ability to represent and extract form data human intelligible and understandable, transparent linguistic information in the form of IF ...THEN rules.

In addition, since mid-1970s (Mamdani [7] or Zadeh-Mamdani [5]) and since mid-1980s (TakagiSugeno [8]) FRB systems started to be developed and are now widely applied. Although, there are other types of fuzzy systems (relational [9], etc.) one particular type of FRB systems which we introduced recently with one of the pioneers of fuzzy sets theory, Professor Ronald Yager [10] called AnYa offers a great potential, specifically to address the issue of design of the fuzzy sets. While, both Mamdani-Zadeh and Takagi-Sugeno type of FRB share the exact same antecedent part (the IF) and only (although significantly) differ by the consequent (THEN) part, theYa type FRB has a quite different antecedent (IF) part. The main issue in the design of the fuzzy sets and systems is the very fundamental one - the membership function by which they are defined in first place. It is practically very difficult and controversial to define membership functions both form experts and from data. This is also related to the more general issue of assumptions made and handcrafting which machine learning (including statistical methods) are facing and is now hotly researched.

Recently, we proposed a new approach [11], which leads to a new form of fuzzy sets and systems empirical fuzzy sets and FRB systems ( $\varepsilon F S$ and $\varepsilon F R B$, respectively). $\varepsilon F S$ and $\varepsilon F R B$ allow preserving the subjective specifics that fuzzy sets and systems are strong with. At the same time, $\varepsilon F S$ and $\varepsilon F R B$ can benefit from the vast amount of data that may be available. For example, $\varepsilon F S$ and $\varepsilon F R B$ still allow extracting expert knowledge by questionnaires or other forms, but will make this much more easy for the expert and not ambiguous (the experts is not asked to define membership values or parameters, but only (optionally) the labels/names of the linguistic terms, classes (if any). For example, if we chose a car, we can simply say which one we like (or possibly how much), but we do not need to specify why or define per feature (price, max speed, etc.).

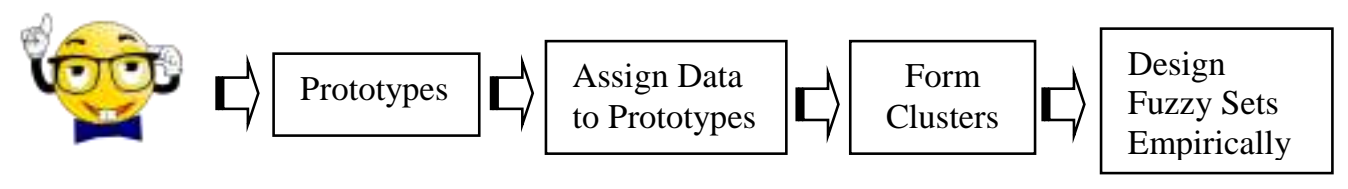

Fig. 3 Subjective design of empirical fuzzy sets 
Moreover, with these new type of $\varepsilon \mathrm{FS}$ and $\varepsilon \mathrm{FRB}$ one can tackle heterogeneous data and combine categorical (e.g. gender, occupation, number of doors) with continuous variables like price, max speed, size, etc.

However, $\varepsilon F S$ and $\varepsilon F R B$ can also be designed in a data-driven manner, see Fig. 4.

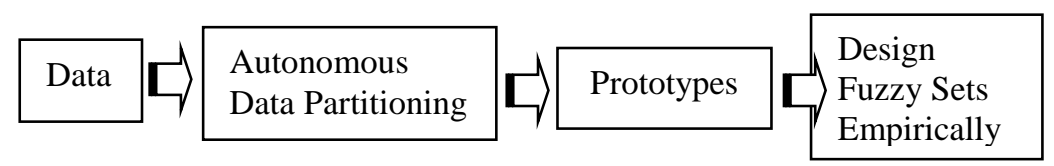

Fig. 4 data-driven design of empirical fuzzy sets

More details are provided in [6] and [11].

On the basis of $\varepsilon F S$ and $\varepsilon F R B$ one can build empirically fuzzy classifiers ( $\varepsilon F$ Classifiers), predictors ( $\varepsilon F$ Predictors), controllers ( $\varepsilon F$ Controllers), recommender systems, etc. Moreover, these can be evolving, not just fixed structure. This will allow studying the dynamic changes in human preferences as well as to build more efficient recommender systems where the only necessary input form the users is the preference ("likes" or "retweets" or "clicks").

\section{References:}

[1] L. A. Zadeh, Fuzzy Sets, Information and Control, 8(3): 338-353, 1965.

[2] Qiangfu Zhao, Dimitar Filev - A Pioneer in Car Intellgence, SMC Magazine, 2018, to appear.

[3] K. Hornik, Approximation Capabilities of Multilayer Feedforward Networks, Neural Networks, 4(2), 251257, 1991.

[4] L. X. Wang, J. M. Mendel, Fuzzy basis functions, universal approximation, and orthogonal least-squares learning, IEEE Transactions on Neural Networks, 1992 3(5):807-814

[5] L A. Zadeh, The Concept of Linguistic variable and it applications to approximate reasoning - II, Information Sciences, 8(4): 301-357, 1975

[6] P. Angelov, X. Gu, Empirical Approach to Machine Learning, ch.5, Springer, 2018.

[7] Mamdani, E.H. and S. Assilian, "An experiment in linguistic synthesis with a fuzzy logic controller," International Journal of Man-Machine Studies, Vol. 7, No. 1, pp. 1-13, 1975.

[8] T. Takagi and M. Sugeno, Fuzzy identification of systems and its applications to modeling and control, IEEE Transactions on Systems, Man, and Cybernetics, 15(1):16-12, 1985.

[9] W. Pedrycz, An Identification algorithm in fuzzy relational systems, Fuzzy Sets and Systems, 13(2): 153-167, 184.

[10] P. Angelov and R. Yager, A new type of simplified fuzzy rule-based system, International Journal on General Systems, 41(2):163-185, 2011.

[11] P. Angelov, X. Gu, Empirical Fuzzy Sets, International Journal of Intelligent Systems, DOI: 10.1002/int.21935, 2017

[12] P. Angelov, S. Sotirov, Imprecision and uncertainty in information representation and processing: new tools based on intuitionistic fuzzy sets and generalized nets, Springer, 2015. 